\title{
Influence of Single Nucleotide Polymorphisms in COMT, MAO-A and BDNF Genes on Dyskinesias and Levodopa Use in Parkinson's Disease
}

\author{
Perdita Cheshire $^{a}$ Kelly Bertram ${ }^{b}$ Helen Ling ${ }^{\text {e, }}$ Sean S. O'Sullivan ${ }^{\text {f, }}$ \\ Glenda Halliday ${ }^{d}$ Catriona McLean ${ }^{c}$ Jose Bras ${ }^{g}$ Tom Foltynie ${ }^{\text {h }}$ \\ Elsdon Storey ${ }^{a, b}$ David R. Williams ${ }^{a, b}$ \\ a Department of Medicine (Neuroscience), Monash University (Alfred Hospital), b Neurology Department, \\ Alfred Hospital, and ${ }^{\mathrm{C}}$ Anatomical Pathology, Alfred Hospital, Melbourne, Vic., and ${ }^{\mathrm{d}}$ Neuroscience Research \\ Australia and University of New South Wales, Sydney, N.S.W., Australia; ${ }^{\mathrm{e}}$ Queen Square Brain Bank for Neurological \\ Disorders, ${ }^{\text {f }}$ eta Lila Weston Institute, ${ }^{9}$ Department of Molecular Neuroscience, and h Sobell Department of \\ Motor Neuroscience and Movement Disorders, Institute of Neurology, University College London, London, UK; \\ 'Neurosciences Department, Cork University Hospital, Cork, Ireland
}

\section{Key Words}

Parkinson's disease - Levodopa-induced dyskinesias . Catechol-O-methyltransferase $\cdot$ Monoamine oxidase A . Brain-derived neurotrophic factor

\section{Abstract \\ Background: Clinical heterogeneity in the development of levodopa-induced dyskinesias (LID) suggests endogenous factors play a significant role in determining their overall prevalence. Objective: We hypothesised that single nucleo- tide polymorphisms (SNPs) in specific genes may result in a clinical phenotype conducive to an increased risk of LID. Methods: We examined the influence of SNPs in the catechol- O-methyltransferase (COMT), monoamine oxidase A (MAO-A) and brain-derived neurotrophic factor (BDNF) genes on LID in a cohort of 285 pathologically confirmed Parkinson's disease patients, using data from their complete disease course. $\boldsymbol{R e}$ - sults: Dyskinetic patients demonstrated younger age at dis- ease onset ( 60.3 vs. 66.4 years, $p<0.0001)$, a longer disease duration (17.0 vs. 12.0 years, $p<0.0001$ ) and a higher maxi- mum daily levodopa equivalent dose (LED; 926.7 vs. 617.1}

\begin{tabular}{ll}
\hline KARGER & $\begin{array}{l}\text { (c) } 2013 \mathrm{~S} . \text { Karger AG, Basel } \\
\text { 1660-2854/14/0131-0024\$39.50/0 Oper }\end{array}$ \\
$\begin{array}{l}\text { E-Mail karger@karger.com } \\
\text { www.karger.com/ndd }\end{array}$ & $\begin{array}{l}\text { This is an Open Access article licensed under the terms of the } \\
\text { Creative Commons Attribution-NonCommercial 3.0 Un- } \\
\text { ported license (CC BY-NC) (www.karger.com/OA-license), } \\
\text { applicable to the online version of the article only. Distribu- } \\
\text { tion permitted for non-commercial purposes only. }\end{array}$
\end{tabular}

$\mathrm{mg} /$ day, $\mathrm{p}<0.0001)$ than patients without dyskinesias. No individual SNP was found to influence prevalence or time to onset of dyskinesias, including after adjustment for known risk factors. We observed that patients carrying alleles conferring both high COMT activity and increased MAO-A mRNA expression received significantly higher maximum and mean daily LEDs than those with low enzyme activity/mRNA expression (max LED: $835 \pm 445$ vs. $508 \pm 316 \mathrm{mg} ; \mathrm{p}=0.0056$, mean LED: $601 \pm 335$ vs. $398 \pm 260$ mg; $p=0.025)$. Conclusions: Individual SNPs in BDNF, COMT and MAO-A genes did not influence prevalence or time to onset of dyskinesias in this cohort. The possibility that combined COMT and MAO-A genotype is a significant factor in determining an individual's lifetime levodopa exposure warrants further investigation.

Copyright $\odot 2013$ S. Karger AG, Basel

\section{Introduction}

Levodopa-induced dyskinesias (LID) are a substantial barrier to effective symptomatic management of Parkinson's disease (PD) and occur as a consequence of chronic 
levodopa (L-DOPA) treatment. Despite their high prevalence, therapeutic options are currently limited [1]. Epidemiological evidence suggests that the onset and severity of LID varies considerably, with some patients never developing them despite equivalent treatment regimens. This observation suggests endogenous factors play a role in individual susceptibility to LID, and despite established risk factors for developing LID including younger age at PD onset, higher L-DOPA dose, greater disease severity and longer disease duration [2], there has been little research to date regarding genetic susceptibility to LID.

In this study, we used a candidate gene approach to examine functional single nucleotide polymorphisms (SNPs) in which the cellular consequences of the polymorphism have been identified and are directly relevant to dyskinesias. Specifically, we focused on functional SNPs in genes encoding the catechol-O-methyltransferase (COMT) and monoamine oxidase A (MAO-A) enzymes, both of which catabolise dopamine and are central to the therapeutic response of L-DOPA. A valine to methionine substitution at codon 158 of the COMT gene produces a Met variant that catabolises dopamine up to four times slower than its Val counterpart [3]. Given the overlapping role of MAO with COMT, we anticipated that a similar finding might be observed for the synonymous substitution of $\mathrm{T}$ to $\mathrm{G}$ in exon 8 of the $M A O-A$ gene, which promotes MAO-A mRNA expression [4].

Finally, a valine to methionine substitution at codon 66 of the brain-derived neurotrophic factor $(B D N F)$ gene has been identified as having a putative role in influencing time to onset of dyskinesia in PD [5]. We hypothesised that these polymorphisms, individually or combined, may contribute to the risk of developing dyskinesias in PD.

\section{Patients and Methods}

\section{Case Selection}

We identified 285 pathologically confirmed PD cases from the Australian Brain Bank Network (ABBN), Australia and the Queen Square Brain Bank for Neurological Disorders (QSBB), UK with a history of L-DOPA usage and a disease duration of at least 5 years. Patients were excluded if they had confirmed monogenic PD, early symptom onset ( $\leq 40$ years of age) or late symptom onset $(\geq 80$ years of age). Approval for the collection of brain tissue as well as retention of and access to clinical records was granted by the $\mathrm{Hu}$ man Research Ethics Committee of the University of Melbourne $(\mathrm{ABBN})$ and the London Multi-Centre Research Ethics Committee (QSBB).

Genetic Risk Factors for LID and L-DOPA

Use
Genotyping

DNA was extracted using standard methods (QIAamp DNA Kit, Qiagen) and genotyping was performed via the SEQUENOM ${ }^{\mathrm{TM}}$ genotyping platform at the Australian Genome Research Facility for all Australian cases. Cases from the UK were genotyped for the Val158Met COMT polymorphism (dbSNP rs4680) and the T941G MAO-A polymorphism (rs6323) as per Spencer et al. [6]. The BDNF Val66Met polymorphism (rs6265) was genotyped as per Foltynie et al. [7].

\section{Clinical Data}

A systematic review of patients' medical records was performed by movement disorder specialists (K.B., H.L. and S.O'S.) and data including age at $\mathrm{PD}$ onset, disease duration, prevalence and time of onset of dyskinesia, and dopaminergic medication history were recorded. Levodopa equivalent dose (LED), which accounts for other antiparkinsonian drugs, was calculated as per Tomlinson et al. [8]. An approximation of the cumulative lifetime L-DOPA dose was estimated using previously published methods [9]. Mean daily LED was obtained using the calculated lifetime estimate and adjusting for the number of years of L-DOPA treatment.

\section{Statistical Analysis}

We constructed Kaplan-Meier survival curves, with the timedependent variable set as the first recorded incidence of dyskinesias in dyskinetic patients, or disease duration for patients without dyskinesia. Time zero was set as age of PD onset, as this data was more reliably recorded than age at first L-DOPA administration. We used Cox proportional hazards regression and generalised linear modelling to examine the relationship between genotype and time to onset of LID, adjusting for established risk factors. As the distribution of LED was skewed, we used logarithmic transformation to normalise the distribution prior to linear modelling. Statistical analysis was performed using GraphPad Prism (version 5.0, GraphPad Software, San Diego, Calif., USA) or SPSS (version 20, IBM SPSS, New York, N.Y., USA).

\section{Results}

Patients in our combined cohort demonstrated typical PD demographics, with a mean age of onset of $63.0 \pm 9.2$ years, a mean disease duration of $14.8 \pm 6.4$ years and a mean maximum daily LED of $794.2 \pm 431.6 \mathrm{mg} /$ day. Dyskinesias were reported in $61.3 \%$ of patients. Dyskinetic patients demonstrated established risk factors for dyskinesias, including younger age of PD onset (60.3 vs. 66.4 years, $\mathrm{p}<0.0001)$, a longer disease duration (17.0 vs. 12.0 years, $\mathrm{p}<0.0001)$ and a higher maximum daily LED (926.7 vs. $617.1 \mathrm{mg} /$ day, $\mathrm{p}<0.0001$ ) than patients without dyskinesias.

When patients were stratified according to COMT, $M A O-A$ or $B D N F$ genotype, no individual genotype was found to independently influence the prevalence or time to onset of dyskinesias (fig. 1). Individual genotypes were compared for each gene, and genotypes were pooled to 


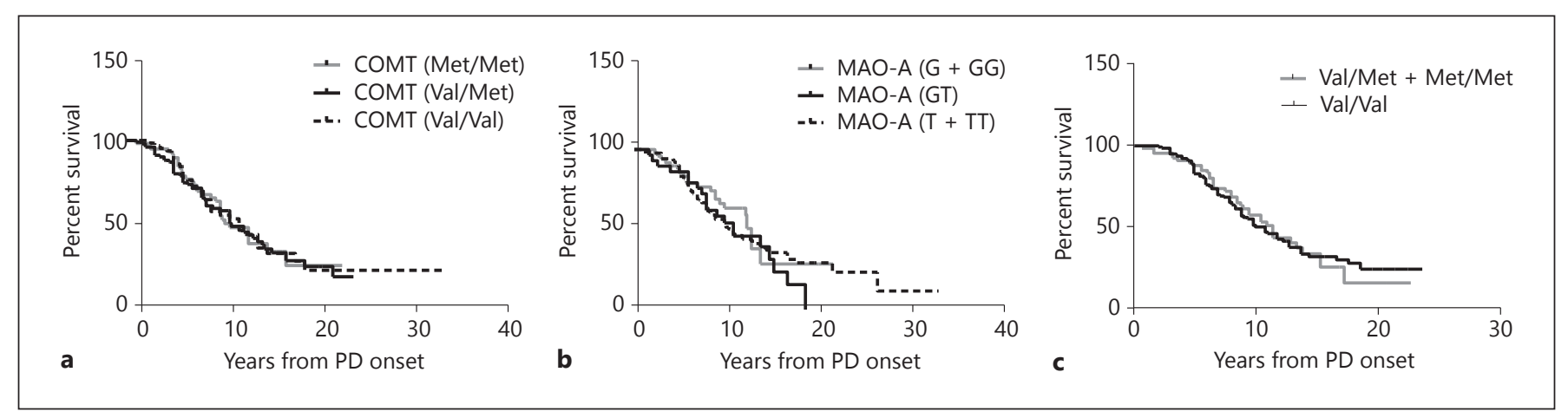

Fig. 1. Survival curves of time to onset of dyskinesia according to COMT (a), MAO-A (b) or BDNF (c) genotype. Kaplan-Meier curves demonstrating duration from $\mathrm{PD}$ onset to first reported incidence of LID in patients stratified according to genotype. For the BDNF Val66Met polymorphism, given the low prevalence of the variant Met allele in European populations, Met/Met homozy- gous patients were pooled with $\mathrm{Val} / \mathrm{Met}$ heterozygotes for statistical analysis. As the MAO-A gene is X-linked, males and females were analysed both separately and with male hemizygotes pooled with female homozygotes to directly compare the effects of T or G allele carriage.

Table 1. Effect of COMT, MAO-A and BDNF genotype on incidence and time to onset of dyskinesias

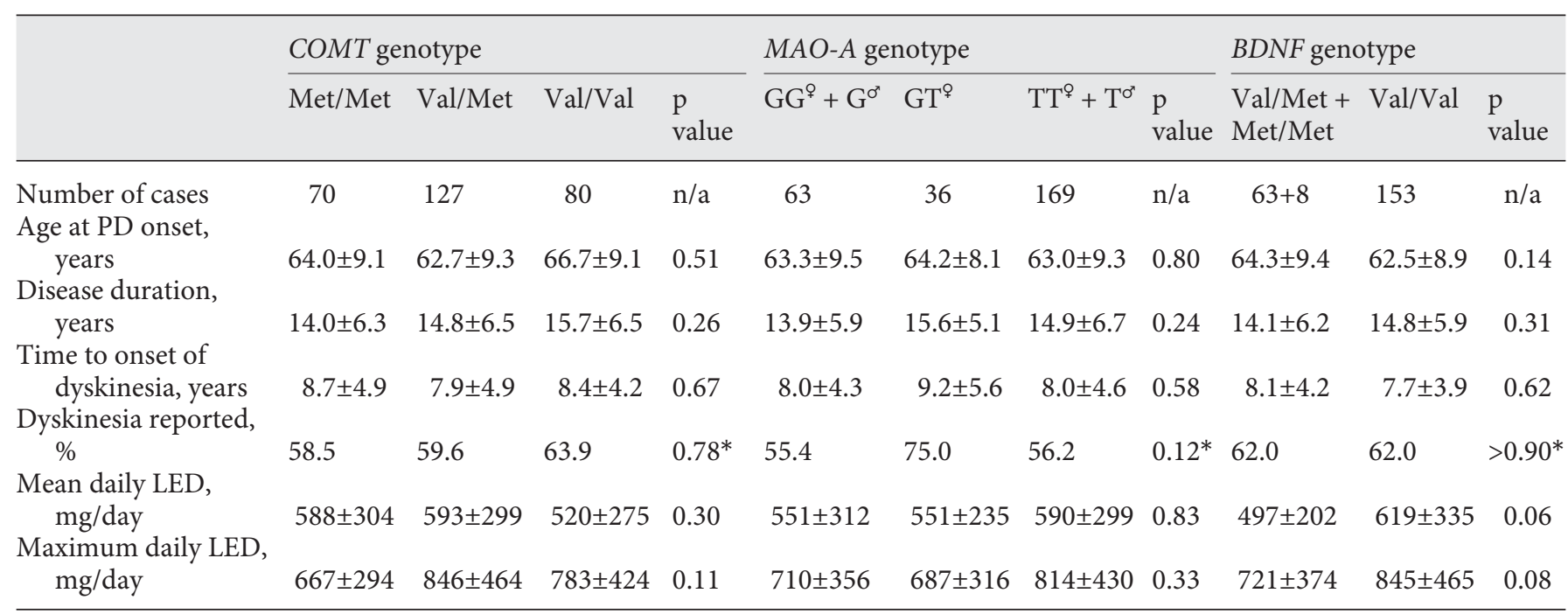

Values are mean \pm SD. Statistical analysis was non-parametric Kruskal-Wallis or Mann-Whitney test. * p value from a $\chi^{2}($ Fisher's exact) test.

examine the effect of homozygosity for either allele. No difference was observed for any clinical feature, including age at onset, disease duration and maximum or mean daily LED (table 1).

We considered that individual functional SNPs may be unlikely to exert a significantly large effect on dyskinesia in isolation, and that given the overlapping function of COMT and MAO-A, double homozygosity for both SNPs may exert a greater effect than homozygosity for either
SNP individually. However, pooling genotypes demonstrated that patients with alleles conferring high COMT activity (Val/Val) and high MAO-A mRNA expression (T males; TT females) did not have a statistically significantly increased prevalence of dyskinesias (58.0 vs. $43.8 \%$, $\chi^{2}=0.62$, d.f. $=1, p=0.43$ ) compared to those with low enzyme activity/mRNA expression. Interestingly, we observed that patients homozygous for high COMT activity and high MAO-A mRNA expression alleles received sig- 
nificantly higher maximum daily LED ( $835 \pm 445 \mathrm{mg})$ than those homozygous for low activity/expression alleles $(508 \pm 316 \mathrm{mg} ; \mathrm{p}=0.0056)$, as well as a higher mean daily $\operatorname{LED}(601 \pm 335$ vs. $398 \pm 260 \mathrm{mg} ; \mathrm{p}=0.0248)$. Generalised linear modelling confirmed the association between pooled COMT and MAO-A genotype and LED, with low activity/expression allele carriers receiving a mean maximum daily LED $43 \%$ lower $(\mathrm{p}=0.003)$ than other genotype groups, after controlling for disease duration.

\section{Discussion}

In this study of pathologically confirmed $\mathrm{PD}$, polymorphisms in COMT, MAO-A and BDNF genes, either individually or combined, had no influence on the prevalence or time to onset of LID (fig. 1). While COMT and BDNF have previously been implicated in the pathophysiology of dyskinesias, MAO-A is a novel candidate. COMT is primarily responsible for the breakdown of LDOPA when DOPA decarboxylase inhibitors are co-administered with L-DOPA, blocking DOPA decarboxylase activity. The COMT rs4680 SNP has been associated with an increased risk of dyskinesias in a recent prospective study [10], although earlier cross-sectional studies failed to find a statistically significant effect [11].

MAO-A has a similar role to COMT in catabolising monoamines, and the T allele of the T941G MAO-A SNP, which confers increased mRNA expression, has been investigated as a risk factor for developing PD [12]. Despite this overlap in function, we found no evidence to suggest that this SNP individually influences dyskinesias. Similarly, met allele carriage of the BDNF Val66Met polymorphism has been associated with an earlier time to onset of dyskinesias [5]; however, our study found no association between BDNF genotype and prevalence or time to onset of dyskinesias, which may be a reflection of different study designs.

Our data suggest a putative relationship between combined COMT and MAO-A genotype and total lifetime LDOPA exposure. Patients with alleles conferring high COMT activity and high MAO-A mRNA expression received significantly higher L-DOPA exposure than low activity/expression carriers. Although these data should be interpreted with caution given the small sample size of this subgroup of double homozygotes, we note that if we reduce our $p$ value to $p<0.01$ to correct for multiple comparisons, our data remain statistically significant.

In a clinical setting, patients with increased COMT enzyme activity and increased MAO-A expression will metabolise L-DOPA faster, which may result in increased wearing off, necessitating higher or more frequent LDOPA doses over the day, resulting in the higher lifetime exposure to L-DOPA observed in our study. This finding warrants replication in a larger cohort, although it is difficult to increase the sample size of a post-mortem cohort without adding cases from additional tissue banks, introducing genetic diversity that can obscure results.

The present study has some important limitations. The data were collected retrospectively using clinical case notes with the implicit assumption that a reasonably consistent threshold would apply for specialists to report dyskinesias. While our patient demographics appear to represent a typical PD population on the basis of age of onset and disease duration, there is likely to be a selection bias in cases submitted for brain banking.

In summary, we find no evidence to suggest the SNPs in COMT, MAO-A or BDNF examined in this study influence the prevalence or time to onset of LID. Our data suggest that combined COMT and MAO-A genotype may influence L-DOPA use, but further studies in different populations are required to clarify this effect.

\section{Acknowledgements}

We would like to thank Catherine Smith, Monash University, for her assistance with statistical modelling and Linda Parsons, QSBB, for her assistance with tissue acquisition. We thank brain donors and their families, without whom this work would not have been possible. This work was funded by the Bethlehem Griffiths Research Foundation, the Brain Foundation, the Wellcome Trust (WTCCC2, grants 084747 and 083948) and Parkinson's UK (K0901). This work was partly undertaken at UCLH/UCL who received a proportion of funding from the Department of Health's NIHR Biomedical Research Centres funding scheme. Support was also received from the Wellcome Trust/MRC Joint Call in Neurodegeneration Award (WT089698) to UCL, the MRC Unit in Dundee and the University of Sheffield. The ABBN is supported by Neuroscience Research Australia, the University of New South Wales and the National Health and Medical Research Council of Australia.

References
1 Fabbrini G, Brotchie JM, Grandas F, Nomoto M, Goetz CG: Levodopa-induced dyskinesias. Mov Disord 2007;22:1379-1389.

-2 Fahn S, Oakes D, Shoulson I, Kieburtz K, Rudolph A, Lang A, et al: Levodopa and the progression of Parkinson's disease. N Engl J Med 2004;351:2498-2508.

-3 Lachman HM, Papolos DF, Saito T, Yu YM, Szumlanski CL, Weinshilboum RM: Human catechol-O-methyltransferase pharmacogenetics: description of a functional polymorphism and its potential application to neuropsychiatric disorders. Pharmacogenetics 1996; 6:243-250. 
4 Pinsonneault JK, Papp AC, Sadee W: Allelic mRNA expression of X-linked monoamine oxidase a (MAOA) in human brain: dissection of epigenetic and genetic factors. Hum Mol Genet 2006;15:2636-2649.

5 Foltynie T, Cheeran B, Williams-Gray CH, Edwards MJ, Schneider SA, Weinberger D, et al: BDNF val66met influences time to onset of levodopa induced dyskinesia in Parkinson's disease. J Neurol Neurosurg Psychiatry 2009; 80:141-144.

6 UK Parkinson's Disease Consortium, Wellcome Trust Case Control Consortium 2, Spencer CC, Plagnol V, Strange A, Gardner M, Paisan-Ruiz C, et al: Dissection of the genetics of Parkinson's disease identifies an additional association $5^{\prime}$ of SNCA and multiple associated haplotypes at $17 \mathrm{q} 21$. Hum Mol Genet 2011;20:345-353.
7 Foltynie T, Lewis SG, Goldberg TE, Blackwell AD, Kolachana BS, Weinberger DR, et al: The BDNF Val66Met polymorphism has a gender specific influence on planning ability in Parkinson's disease. J Neurol 2005;252:833-838.

$>8$ Tomlinson CL, Stowe R, Patel S, Rick C, Gray $\mathrm{R}$, Clarke CE: Systematic review of levodopa dose equivalency reporting in Parkinson's disease. Mov Disord 2010;25:2649-2653.

9 Parkkinen L, O'Sullivan SS, Kuoppamaki M, Collins C, Kallis C, Holton JL, et al: Does levodopa accelerate the pathologic process in Parkinson disease brain? Neurology 2011;77: $1420-1426$.
0 de Lau LM, Verbaan D, Marinus J, Heutink P, van Hilten JJ: Catechol-O-methyltransferase Val158Met and the risk of dyskinesias in Parkinson's disease. Mov Disord 2011;27:132135 .

11 Watanabe M, Harada S, Nakamura T, Ohkoshi N, Yoshizawa K, Hayashi A, et al: Association between catechol-O-methyltransferase gene polymorphisms and wearing-off and dyskinesia in Parkinson's disease. Neuropsychobiology 2003;48:190-193.

12 Williams-Gray C, Goris A, Foltynie T, Compston A, Sawcer S, Barker RA: No evidence for association between an MAOA functional polymorphism and susceptibility to Parkinson's disease. J Neurol 2009;256:132133. 